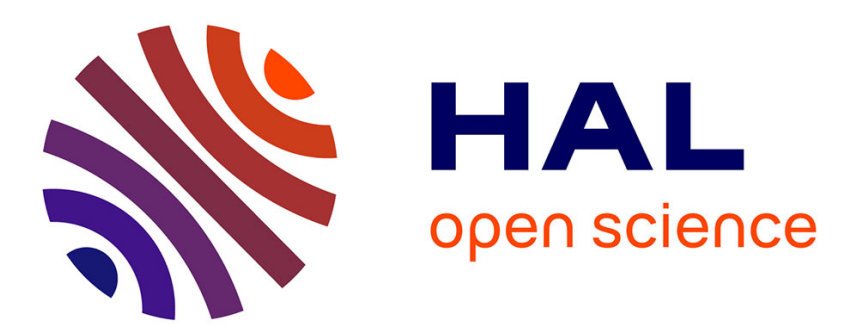

\title{
Nash-MFG equilibrium in a SIR model with time dependent newborn vaccination
}

\author{
Emma Hubert, Gabriel Turinici
}

\section{To cite this version:}

Emma Hubert, Gabriel Turinici. Nash-MFG equilibrium in a SIR model with time dependent newborn vaccination. Ricerche di matematica, 2018, Demographic and temporal heterogeneity in infectious disease epidemiology, 67 (1), pp.227-246. 10.1007/s11587-018-0365-0 . hal-01389584v2

\section{HAL Id: hal-01389584 \\ https://hal.science/hal-01389584v2}

Submitted on 24 Apr 2017

HAL is a multi-disciplinary open access archive for the deposit and dissemination of scientific research documents, whether they are published or not. The documents may come from teaching and research institutions in France or abroad, or from public or private research centers.
L'archive ouverte pluridisciplinaire HAL, est destinée au dépôt et à la diffusion de documents scientifiques de niveau recherche, publiés ou non, émanant des établissements d'enseignement et de recherche français ou étrangers, des laboratoires publics ou privés. 


\title{
NASH-MFG EQUILIBRIUM IN A SIR MODEL WITH TIME DEPENDENT NEWBORN VACCINATION
}

\author{
EMMA HUBERT AND GABRIEL TURINICI
}

\begin{abstract}
We study the newborn, non compulsory, vaccination in a SIR model with vital dynamics. The evolution of each individual is modeled as a Markov chain. His/Her vaccination decision optimizes a criterion depending on the time-dependent aggregate (societal) vaccination rate and the future epidemic dynamics. We prove the existence of a Nash - Mean Field Games equilibrium among all individuals in the population. Then we propose a novel numerical approach to find the equilibrium and test it numerically.
\end{abstract}

\section{INTRODUCTION}

To analyze the impact of societal vaccination policies on epidemic dynamics, one has to distinguish two situations. First, when the vaccination is compulsory or when the public adheres to a very large extent to the official recommendations, the vaccination policy can be supposed implementable in full and the problem can take the form of an optimization problem of costs with respect to benefits (either in terms of money or of medical states, or of both), see for instance [36, 1, 47, 53, 22, 41].

On the contrary, when vaccination is not compulsory or the official recommendations are followed to a lesser extent, the individual choices have to be taken into account. Works in this direction include $[26,12,32,7,8,54,19,18,49,31,21,20,14,13,23$, $24,50,30,56,10,15,48,29,5]$ (see also the recent review [57]). It was shown that this decision depends on the present and past states of the epidemic (see [46]), sometimes on the prediction of its future course (see [50]) and on the individual perception of two costs: a cost $r_{V}$ incurred if the individual vaccinates and a cost $r_{I}$ incurred if the individual is infected; again, the costs are not necessarily expressed in terms of money but can also be medical side effects or general morbidity (see [58, 2, 52] for an introduction on QALY/DALY).

So, on the one hand, each individual chooses according to his/her own perception and interests. His/Her decision, as single individual, cannot influence the societal epidemic dynamics but is influenced by it. Such a situation is called in physical sciences a "mean field" that mediates the interaction between agents. At its turn, the mean field is the aggregation of the decisions of all individuals. An important question is the existence of a stable equilibrium between the individual decisions and the overall observed dynamics. From the mathematical point of view, the formalism is that of a Nash equilibrium in a game with an infinity of players. Recently, such approaches witnessed an important development with the introduction of the Mean Field Games (henceforth MFG) techniques in the works of Lasry and Lions [44, 43, 45] and of Huang, Malhamé and Caines $[38,37]$ (see also [9] and [35] for entry points to the litterature).

Date: April 24, 2017.

Key words and phrases. vaccination; Mean Field Games; SIR model; individual vaccination; vaccination games; vaccination efficacy; Nash equilibrium. 
Within this framework, the contribution presented here is more specifically targeted to analyze newborn vaccination in a SIR model with vital dynamics. Such a model has already been considered in [6] which is the main inspiration and starting point of our work; however in [6] the newborn vaccination rate is supposed constant in time. Our goal is

(1) to show that an equilibrium exists when also considering time depending vaccination rate;

(2) that this is the generic situation;

(3) and to propose a numerical algorithm to compute the equilibrium dynamics (because now the unknown is not just a real number but a real valued function defined on $\mathbb{R}_{+}$).

As a technical remark, our analysis follows the line of thought formalized in [27, 42, 50] with the distinction that here we have no analytic solution and have to resort to more general techniques.

The balance of the paper is the following: in section 2 we introduce the model and see that keeping the vaccination rate constant may not be realistic: several hundreds of years may be required to reach the constant-rate equilibrium, which is not compatible with the individual time span. Nevertheless the long time limit is right and one has only to study the transitory regime. This is done in section 3 , where we prove that the model with a time-varying newborn vaccination rate has an equilibrium. The computation of the equilibrium is then explained in section 4 and numerical simulations are presented to illustrate the results.

\section{Motivation AND MOdEL NOtATions}

2.1. The model and previous works. We consider the SIR model with vital dynamics and newborn vaccination (see $[3,4,22])$ :

$$
\begin{aligned}
& S^{\prime}(t)=\mu(1-p)-\beta S I-\mu S \\
& I^{\prime}(t)=\beta S I-\gamma I-\mu I \\
& R^{\prime}(t)=\gamma I-\mu R \\
& V^{\prime}(t)=\mu p-\mu V,
\end{aligned}
$$

where $\mu$ is the death / birth rate, $\beta$ is the transmission rate, $1 / \gamma$ is the infectious period and $p(t)$ is the vaccine uptake level for the individuals born at time $t$ (assuming, for simplicity, that infection cannot occur before vaccination). In this model $S$ represents the susceptible individuals, $I$ the infected ones, $R$ the recovered and $V$ the vaccinated.

The initial conditions are set once for all:

$$
S(0)=S_{0}, I(0)=I_{0}, R(0)=1-S(0)-I(0), V(0)=0 .
$$

Remark 2.1. The population is supposed at equilibrium i.e., the birth rate equals the death rate; the model can be adapted in a straightforward manner to treat the general case.

The model has already been considered in [6], but there, $p$ is assumed constant. It was proved that, if the individuals act according to their own self-interest (see later in section 2.4 the precise definition of the individual decision criterion), then the overall dynamics will converge, in some unknown time, to an endemic equilibrium characterized by some level of susceptibles $S^{\infty}$, of infected $I^{\infty}$ and an equilibrium vaccination rate 
(stable Nash equilibrium), denoted $p_{\text {csne }}$. Let us set $R_{0}=\beta /(\gamma+\mu)$ and $r=r_{V} / r_{I}$ (the quotient of the costs of the vaccine and of the illness).

In order to see a numerical illustration, we take the relevant situation when $R_{0}(1-r)>$ 1 ; then the equilibrium vaccination is $p_{c s n e}=1-\frac{1}{R_{0}(1-r)}$ and the system converges to the endemic equilibrium:

$$
S^{\infty}=1 / R_{0}, I^{\infty}=\frac{\mu}{\gamma+\mu} \frac{r}{R_{0}(1-r)}, R^{\infty}=\frac{\gamma}{\mu} I^{\infty}, V^{\infty}=p_{\text {csne }} .
$$

For the parameters in [6], even if we start close to the theoretical equilibrium, the model may take long to converge (see figure 1). Therefore, even if the long time individual vaccination behavior is known, this still does not provide information on the transitory dynamics i.e., on the evolution towards this equilibrium, because the individuals are not expected to plan over such a long horizon. On the other hand, a time dependent vaccination rate results in faster dynamics and lowers the time-horizon of the individual decision.. Of course, the precise values of the time horizon depends on the specific parameters of the model. See also section 4.3 and figure 5 for additional advantages of a model with time dependent vaccination rate.

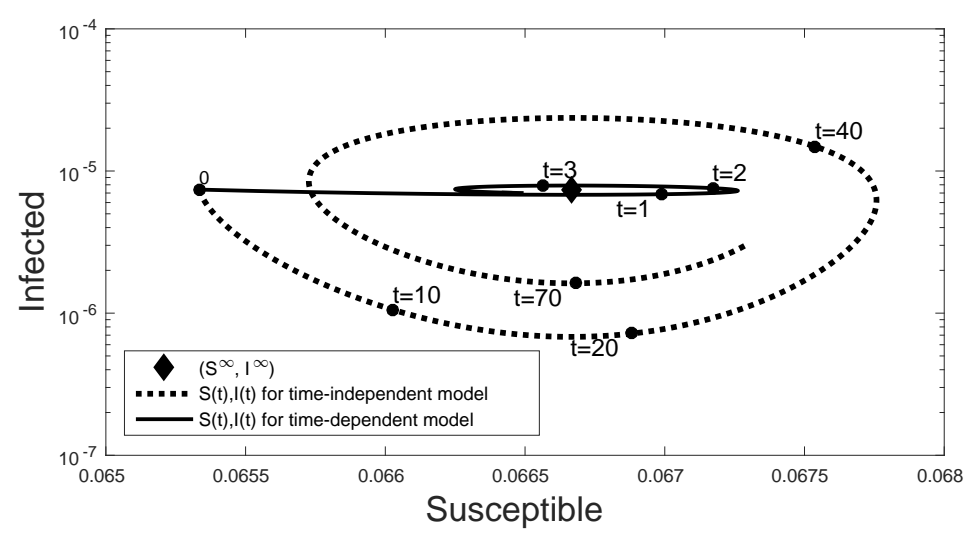

Figure 1. Dotted line: evolution of the susceptibles and infected classes in the time independent vaccination rate model [6] with parameters : $\mu=1 / 80, R_{0}=15, \gamma / \mu=1000, \beta=R_{0} *(\gamma+\mu), r=0.1$, $T=80$ (years) and initial parameters $S_{0}=6.53 \%, I_{0}=7.4 e-6$. For these parameters $p_{\text {csne }}=0.9259$ is a convergently stable Nash equilibrium (within class of the time independent vaccination rates) with limit state $S^{\infty}=6.6 \%, I^{\infty}=7.4 e-6$. More than a hundred years is required in order for the long time limit to be reached. Solid line: the evolution of the susceptibles and infected classes in the time dependent vaccination rate model (2.8)-(2.11) for a total time $T=5$; faster convergence towards $\left(S^{\infty}, I^{\infty}\right)$ is observed.

2.2. First notations. We denote the set of all admissible vaccination strategies as:

$$
\mathcal{P}=\left\{p: \mathbb{R}_{+} \mapsto[0,1], p \text { measurable }\right\} .
$$

The interpretation is that the individual born at $t$ will be vaccinated with probability $p(t)$; note that this implies that we are dealing with mixed strategies (see [28] for some discussions on mixed versus pure strategies). 
As a technical remark, note that the system (2.1)-(2.4) does not satisfy the usual hypothesis for the existence of a solution because $p$ may have no regularity at all (other than being bounded and measurable). To show that a solution exists, one has to write the system in the form:

$$
\begin{aligned}
& S^{\prime}(t)=\mu(1-\dot{u})-\beta S I-\mu S \\
& I^{\prime}(t)=\beta S I-\gamma I-\mu I \\
& R^{\prime}(t)=\gamma I-\mu R \\
& V^{\prime}(t)=\mu \dot{u}-\mu V
\end{aligned}
$$

where we used the cummulative vaccination function $u(t)=\int_{0}^{t} p(\tau) d \tau$ which belongs to the set:

$$
\mathcal{C}=\{u:[0, \infty[\rightarrow \mathbb{R}|u(0)=0, \forall b \geq a \geq 0:| u(a)-u(b)|\leq| a-b \mid, u(b) \geq u(a)\}
$$

Here, we replaced $p$ by $\dot{u}$ which is a Clarke generalized gradient of $u$ (see appendix A and $[17,16]$; we also denote $p \in \bar{\partial} u$ or $p \in \dot{u}$ ). In this form, the results from [11] apply and allow to estimate the solution in terms of the $L^{1}$ norm of $u$.

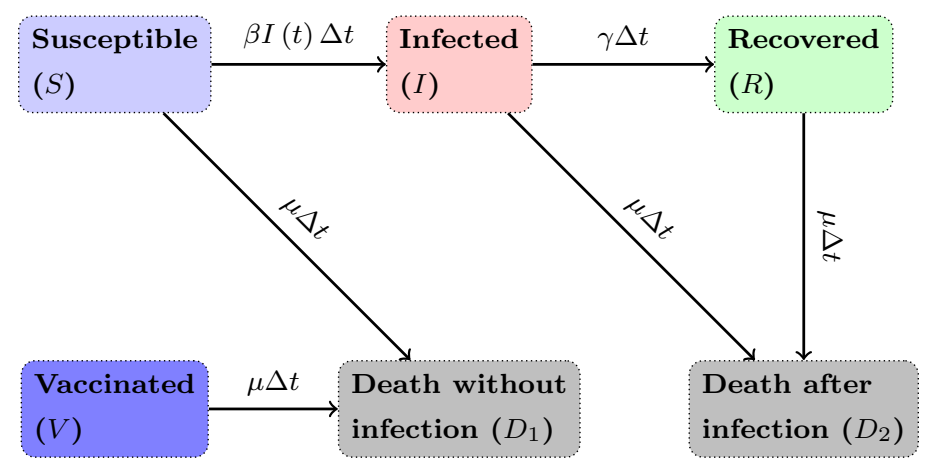

Figure 2. Individual model is a continuous time non-homogeneous Markov chain.

Remark 2.2. The model can accommodate the situation when vaccination may occur later during the lifetime of the individual (i.e., not necessarily at birth). In this case the term $\mu p$ in equations (2.1) and (2.4) is to be replaced by the vaccination rate and more importantly the sets $\mathcal{P}$ and $\mathcal{C}$ are to be adapted in order to ensure $S(t) \geq 0$ at all times.

Individuals seek to optimize an individual cost function. In order to define it, we have to consider the individual dynamics (see figure 2 for an illustration). It takes the form of a controlled Markov chain with several states, Susceptible (S), Vaccinated (V), Recovered (R), Infected (I), Death without any infection $\left(D_{1}\right)$ and Death after infection $\left(D_{2}\right)$. 
The Markov chain of the individual born in $t \geq 0$, whose state at time $\tau \geq t$ is denoted $M_{\tau}^{t}$, is described in terms of the passage probabilities :

$$
\begin{aligned}
\mathbb{P}\left(M_{\tau+\Delta \tau}^{t}=S \mid M_{\tau}^{t}=S\right) & =1-\beta I(\tau) \Delta \tau-\mu \Delta \tau+o(\Delta \tau) \\
\mathbb{P}\left(M_{\tau+\Delta \tau}^{t}=I \mid M_{\tau}^{t}=S\right) & =\beta I(\tau) \Delta \tau+o(\Delta \tau) \\
\mathbb{P}\left(M_{\tau+\Delta \tau}^{t}=D_{1} \mid M_{\tau}^{t}=S\right) & =\mu \Delta \tau+o(\Delta \tau) \\
\mathbb{P}\left(M_{\tau+\Delta \tau}^{t}=I \mid M_{\tau}^{t}=I\right) & =1-\gamma \Delta \tau-\mu \Delta \tau+o(\Delta \tau) \\
\mathbb{P}\left(M_{\tau+\Delta \tau}^{t}=R \mid M_{\tau}^{t}=I\right) & =\gamma \Delta \tau+o(\Delta \tau) \\
\mathbb{P}\left(M_{\tau+\Delta \tau}^{t}=D_{2} \mid M_{\tau}^{t}=I\right) & =\mu \Delta \tau+o(\Delta \tau) \\
\mathbb{P}\left(M_{\tau+\Delta \tau}^{t}=R \mid M_{\tau}^{t}=R\right) & =1-\mu \Delta \tau+o(\Delta \tau) \\
\mathbb{P}\left(M_{\tau+\Delta \tau}^{t}=D_{2} \mid M_{\tau}^{t}=R\right) & =\mu \Delta \tau+o(\Delta \tau) \\
\mathbb{P}\left(M_{\tau+\Delta \tau}^{t}=V \mid M_{\tau}^{t}=V\right) & =1-\mu \Delta \tau+o(\Delta \tau) \\
\mathbb{P}\left(M_{\tau+\Delta \tau}^{t}=D_{1} \mid M_{\tau}^{t}=V\right) & =\mu \Delta \tau+o(\Delta \tau) \\
\mathbb{P}\left(M_{\tau+\Delta \tau}^{t}=D_{1} \mid M_{\tau}^{t}=D_{1}\right) & =1 \\
\mathbb{P}\left(M_{\tau+\Delta \tau}^{t}=D_{2} \mid M_{\tau}^{t}=D_{2}\right) & =1
\end{aligned}
$$

Since the individual born in $t$ vaccinates with probability $p(t)$, the initial state $M_{t}^{t}$ follows the Bernoulli distribution :

$$
M_{t}^{t}= \begin{cases}S & \text { with probability } 1-p(t) \\ V & \text { with probability } p(t) .\end{cases}
$$

2.3. The probability of infection. In order to explain the choice of an individual (to vaccinate or not and with which probability), we have to compute the probability of being infected. Let $\phi_{I}^{u}($.$) be a function from \mathbb{R}_{+}$to $[0,1]$ such that, for any $t \in \mathbb{R}_{+}$, $\phi_{I}^{u}(t)$ is the probability of infection during the life of an individual, born in $t$ and not vaccinated, when the rest of the population follows the vaccination function $p \in \dot{u}$. In mathematical terms, for any individual born in $t \geq 0$,

$$
\phi_{I}^{u}(t)=\mathbb{P}\left(\exists \tau \geq t \text { such that } M_{\tau}^{t}=I \mid M_{t}^{t}=S\right) .
$$

We can prove (see Section B) that:

$$
\phi_{I}^{u}(t)=1-\int_{t}^{+\infty} \mu \exp \left(-\int_{t}^{\tau}\left(\mu+\beta I^{u}(s)\right) d s\right) d \tau
$$

Moreover

$$
\phi_{I}^{u}(t)=1-\frac{F_{I}^{u}(\infty)-F_{I}^{u}(t)}{f_{I}^{u}(t)}, \phi_{I}^{u}(0)=1-F_{I}^{u}(\infty),
$$

where the functions $f_{I}^{u}(t)$ and $F_{I}^{u}(t)$ satisfy, for $t \geq 0$, the following differential equations:

$$
\begin{aligned}
\frac{d}{d t} f_{I}^{u}(t) & =-\left(\mu+\beta I^{u}(t)\right) f_{I}^{u}(t), \\
\frac{d}{d t} F_{I}^{u}(t) & =\mu f_{I}^{u}(t)
\end{aligned}
$$

with initial conditions:

$$
f_{I}^{u}(0)=1, F_{I}^{u}(0)=0 .
$$


2.4. The individual cost. Recall that $r_{I}$ is the individual cost of the illness and $r_{V}$ the vaccination cost. We suppose that $r_{I}>r_{V}$ and work with $r=r_{V} / r_{I}$. Suppose that the entire population follows the vaccination schedule $\dot{u}$, the expected cost for any individual born in $t$ with vaccination probability $p_{I}(t)$ is:

$$
p_{I}(t) r_{V}+\left(1-p_{I}(t)\right) r_{I} \phi_{I}^{u}(t)=p_{I}(t) r_{V}-p_{I}(t) r_{I} \phi_{I}^{u}(t)+r_{I} \phi_{I}^{u}(t) .
$$

We consider that all individual are rational and minimize their cost. Given the vaccination $\dot{u}$ of the population, the minimization problem for an individual born in $t$ is:

$$
\min _{p_{I}(t) \in[0,1]}\left\{p_{I}(t)\left(r_{V}-r_{I} \phi_{I}^{u}(t)\right)\right\}
$$

2.5. Nash equilibrium. A set of strategies is a Nash equilibrium if no individual can do strictly better by unilaterally changing his/her strategy. The goal is to prove that, in our model, there exists a Nash equilibrium. It is equivalent to finding a fixed point of the function which associate to any element in $\mathcal{P}$ all strategies in $\mathcal{P}$ which minimize the cost of an individual (supposing everybody else uses as strategy the argument of the function). See section 3.3 for a precise mathematical definition.

\section{EXISTENCE OF AN EQUILIBRIUM}

Set $\mathcal{L}=\mathcal{C}^{0}([0,+\infty[, \mathbb{R})$, the set of continuous functions in $[0,+\infty[$ with real values. For any $p \in \mathcal{P}$, the function $t \mapsto u(t)=\int_{0}^{t} p(s) d s$ belongs to the set $\mathcal{C} \subset \mathcal{L}$ defined by equation (2.12).

Conversely, for any $u \in \mathcal{C}$, the set $\dot{u}$ of its Clarke generalized gradients [17] is a subset of $\mathcal{P}$. In particular $\forall \tau \geq 0: u(\tau)=\int_{0}^{\tau} \dot{u}(s) d s$.

Finding a Nash equilibrium in our model is equivalent to finding a fixed point of the following multivalued correspondence $\mathcal{T}: u \in \mathcal{C} \mapsto \mathcal{T}(u) \in \mathcal{P}(\mathcal{C})$ (here $\mathcal{P}(\mathcal{C})$ is the ensemble of subsets of $\mathcal{C}$ ) where

$$
\mathcal{T}(u)=\left\{v \in \mathcal{C} \mid \exists p \in \dot{v}: p(\tau)\left(r_{V}-r_{I} \phi_{I}^{u}(\tau)\right) \leq q(\tau)\left(r_{V}-r_{I} \phi_{I}^{u}(\tau)\right), \forall q \in \mathcal{P}, \forall \tau \geq 0\right\} .
$$

One of the main results of this work is the proof of existence of a Nash-MFG equilibrium and is formalized in the following

Theorem 3.1. The model (2.1)-(2.4) admits at least one Nash equilibrium.

Proof We use the following fixed-point theorem (see [33, 25]):

Theorem 3.2 (Kakutani-Fan-Glicksberg). Let $\mathcal{L}$ be a locally convex topological linear space and $\mathcal{C}$ a compact convex set in $\mathcal{L}$. Let $\mathcal{R}(\mathcal{C})$ be the family of all closed convex (non empty) subsets of $\mathcal{C}$. Then, for any upper semi-continuous point to set correspondence $\mathcal{T}$ from $\mathcal{C}$ to $\mathcal{R}(\mathcal{C})$, there exists a point $x_{0} \in \mathcal{C}$ such that $x_{0} \in \mathcal{T}\left(x_{0}\right)$.

The assumptions of the theorem will be checked one by one in the rest of this Section.

3.1. The space $\mathcal{L}$. In order to apply the previous theorem, we need to define a topology on $\mathcal{L}$. Let $K_{n}=[0, n]$ be a sequence of compact sets in $\mathbb{R}_{+}$, we consider the countable family of seminorms $\eta_{n}(f)=\sup _{x \in K_{n}}|f(x)|$ for $f \in \mathcal{L}$. This family of seminorms is well defined, separated and induce the compact-open topology. The set $\mathcal{L}$ is completely metrizable with the distance $d$, which induce the same topology :

$$
d(f, g)=\sum_{n \in \mathbb{N}^{*}} \frac{\eta_{n}(f-g)}{2^{n}\left(1+\eta_{n}(f-g)\right)} .
$$


The set $\mathcal{L}$ equipped with this family of seminorms is a locally convex topological vector space. Hence, $\mathcal{L}$ satisfies the assumptions of the theorem.

3.2. The subset $\mathcal{C}$. We have to prove that $\mathcal{C}$, defined by $(2.12)$, is a compact convex set in $\mathcal{L}$. Straightforward computations show that $\mathcal{C}$ is convex. In order to prove that $\mathcal{C}$ is a compact set, we will use the following result, which is a form of $[39$, Theorem 17 , page 233] :

Theorem 3.3 (Arzela-Ascoli). Let $X$ be a separated topological space and $Y$ be a metric space. Let $\mathcal{C}$ be a subset of $\mathcal{C}^{0}(X, Y)$ such that :

- $\mathcal{C}$ is equicontinuous;

- $\mathcal{C}$ is closed;

- for all $x$ in $X$, the set $\mathcal{C}(x)=\{u(x) \mid u \in \mathcal{C}\}$ is relatively compact in $Y$.

Then the subset $\mathcal{C}$ is compact in $\mathcal{C}^{0}(X, Y)$ for the compact-open topology.

First of all, $X=\mathbb{R}_{+}$is a separated topological space and $Y=\mathbb{R}$ is a metric space. For the first point, the functions in $\mathcal{C}$ are Lipschitz functions (with Lipschitz constant equal to 1 ), hence the space $\mathcal{C}$ is equicontinuous.

In order to prove the second assumption on $\mathcal{C}$, let $\left(u_{k}\right)_{k \geq 1} \subset \mathcal{C}$ be a sequence converging to a limit $u$ in the topology of $\mathcal{L}$. First, $u$ is an uniform limit (on compact sets) of a sequence of continuous functions, hence $u$ is continuous. Secondly, $u(0)=\lim _{k \rightarrow \infty} u_{k}(0)=0$ because $\left(u_{k}\right)_{k \geq 1}$ is converging uniformly on compact sets to $u$, hence is also simply converging. Finally, $u$ is a Lipschitz continuous function : indeed, let $a, b \in \mathbb{R}_{+}$, we have :

$$
\begin{aligned}
|u(a)-u(b)| & =\left|u(a)-u_{k}(a)+u_{k}(a)-u_{k}(b)+u_{k}(b)-u(b)\right| \\
& \leq\left|u(a)-u_{k}(a)\right|+\left|u_{k}(a)-u_{k}(b)\right|+\left|u_{k}(b)-u(b)\right|
\end{aligned}
$$

The first and the last members are converging to zero when $k$ is converging to $+\infty$. The second member can be bounded by $|a-b|$ because for all $k, u_{k} \in \mathcal{C}$, hence $u_{k}$ is Lipschitz. Thus, the limit $u$ of the sequence $\left(u_{k}\right)$ is in the subset $\mathcal{C}$, hence $\mathcal{C}$ is closed.

Finally, for all $x$ in $\mathbb{R}_{+}$, we have to show that the set $\mathcal{C}(x)=\{u(x) \mid u \in \mathcal{C}\}$ is relatively compact in $\mathbb{R}$. As $\mathcal{C}(x)$ is a subset of $\mathbb{R}$, we just have to prove that $\mathcal{C}(x)$ is bounded. Let $u(x) \in \mathcal{C}(x)$ :

$$
|u(x)|=|u(x)-u(0)| \leq|x-0|=|x| .
$$

Hence, for all $x \in \mathbb{R}_{+}$, the set $\mathcal{C}(x)=\{u(x) \mid u \in \mathcal{C}\}$ is bounded, thus relatively compact, in $\mathbb{R}$. By applying the previous theorem, the subset $\mathcal{C}$ is compact in $\mathcal{L}=\mathcal{C}^{0}([0,+\infty[, \mathbb{R})$ for the compact-open topology.

3.3. The mapping $\mathcal{T}$. Recall that the mapping $\mathcal{T}: u \in \mathcal{C} \mapsto \mathcal{T}(u) \in \mathcal{P}(\mathcal{C})$ is such that $\mathcal{T}(u)$ is defined by equation 3.1 .

For given $u \in \mathcal{C}$, and $v \in \mathcal{T}(u)$, then there exists $p \in \mathcal{P} \cap \dot{v}$ such that, for any $t \geq 0$ :

$$
\begin{cases}p(t)=0 & \text { if } \phi_{I}^{u}(t)<r \\ p(t) \text { arbitrary in }[0,1] & \text { if } \phi_{I}^{u}(t)=r \\ p(t)=1 & \text { if } \phi_{I}^{u}(t)>r\end{cases}
$$

We need to show that $\mathcal{T}$ is with values in $\mathcal{R}(\mathcal{C})$, the family of all closed convex (non empty) subsets of $\mathcal{C}$, and that $\mathcal{T}$ is an upper semi-continuous point to set correspondence. 
3.3.1. The set $\mathcal{T}(u)$ is closed. Let $u$ in $\mathcal{C}$ and $\left(v_{n}\right)_{n \geq 1}$ be a sequence in $\mathcal{T}(u)$ such that $v_{n}$ converges in $\mathcal{L}$ to a limit $v$. The sequence $\left(v_{n}\right)_{n \geq 1} \subset \mathcal{T}(u)$ is included in the compact set $\mathcal{C}$, hence $v \in \mathcal{C}$. Moreover, for all $n \in \mathbb{N}$, there exists $p_{n}$ associated to $v_{n}$ in the sense of relation (3.1). Let us consider two cases :

(1) If $\phi_{I}^{u}\left(t_{0}\right)<r$ for $t_{0} \geq 0$ : since $\phi_{I}^{u}(\cdot)$ is continuous, there exists $\epsilon>0$ such that $\phi_{I}^{u}(\cdot)<r$ on $\left.V_{t_{0}, \epsilon}=\right] t_{0}-\epsilon, t_{0}+\epsilon\left[\right.$. Hence, on $V_{t_{0}, \epsilon}, p_{n}=0$, i.e. $v_{n}=c s t$. Since $v_{n}$ converges uniformly to $v$ on $V_{t_{0}, \epsilon}, v=c s t$ on $V_{t_{0}, \epsilon}$. Hence, for any $p \in \dot{v}$ we have $p=0$ on $V_{t_{0}, \epsilon}$ and in particular $p\left(t_{0}\right)=0$.

(2) If $\phi_{I}^{u}\left(t_{0}\right)>r$ for $t_{0} \geq 0$ : in this case, we can construct a neighborhood $V_{t_{0}, \epsilon}$ with $p_{n}=1$ on $V_{t_{0}, \epsilon}$ i.e., for all $t \in V_{t_{0}, \epsilon}, v_{n}(t)-v_{n}\left(t_{0}-\epsilon\right)=t-\left(t_{0}-\epsilon\right)$. By uniform convergence on compact sets, $v(t)-v\left(t_{0}-\epsilon\right)=t-\left(t_{0}-\epsilon\right)$, hence, for any $p \in \dot{v}$ we have $p=1$ on $V_{t_{0}, \epsilon}$.

In both cases, we showed that for any $p \in \dot{v}$ we have $p=0$ when $\phi_{I}^{u}(\cdot)<r$ and $p=1$ when $\phi_{I}^{u}(\cdot)>r$. Recall that $\phi_{I}^{u}\left(t_{0}\right)=r$ for $t_{0} \geq 0$ does not impose and constraint on $p$ which can be chosen arbitrary in $[0,1]$. Hence, $p$ and $v$ satisfy the relation (3.1) and thus $v \in \mathcal{T}(u)$.

3.3.2. $\mathcal{T}$ has values in $\mathcal{R}(\mathcal{C})$. Let $u$ in $\mathcal{C}$, we need to show that $\mathcal{T}(u)$ is a closed convex (non empty) subset of $\mathcal{C}$. By the Section 3.3.1, $\mathcal{T}(u)$ is a closed subset of $\mathcal{C}$. For the convexity, let $v, w \in \mathcal{T}(u)$ and $\alpha \in[0,1]$. There exist $p_{v}$ and $p_{w}$ associated respectively to $v$ and $w$, in the sense of relation (3.1). Denote $z=\alpha v+(1-\alpha) w$. Let us consider two cases :

(1) If $\phi_{I}^{u}\left(t_{0}\right)<r$ for $t_{0} \geq 0$ : since $\phi_{I}^{u}(\cdot)$ is continuous, there exist $\epsilon$ such that $\phi_{I}^{u}()<$. $r$ on $\left.V_{t, \epsilon}=\right] t-\epsilon, t+\epsilon\left[\right.$. Hence, on $V_{t, \epsilon}, p_{v}=p_{w}=0$, thus $v$ and $w$ are constant on $V_{t, \epsilon}$. This implies that $z$ is also constant. Hence, $p_{z}=\alpha p_{v}+(1-\alpha) p_{w} \in \dot{z}$ is such that $p_{z}=0$ on $V_{t, \epsilon}$.

(2) If $\phi_{I}^{u}\left(t_{0}\right)>r$ for $t_{0} \geq 0$ : in this case, on some $V_{t, \epsilon}$ we have $p_{v}=p_{w}=1$, i.e. for all $\tau \in V_{t, \epsilon}$ :

$$
v(\tau)-v(t-\epsilon)=\tau-(t-\epsilon), w(\tau)-w(t-\epsilon)=\tau-(t-\epsilon) .
$$

Hence $z(\tau)-z(t-\epsilon)=\tau-(t-\epsilon)$. Thus, $p_{z}=1$ on $V_{t, \epsilon}$.

Using the same arguments than those given in the Section 3.3.1, $z \in \mathcal{T}(u)$.

3.3.3. $\mathcal{T}$ is upper semi-continuous. Since $\mathcal{L}$ is metrizable and $\mathcal{T}(u)$ is a compact set (closed and included in the compact set $\mathcal{C}$ ), in order to prove that the mapping $\mathcal{T}$ is upper semi-continuous, we can use the definition in terms of limits of sequences.

Let $\left(u_{n}\right)_{n \geq 1}$ be a sequence in $\mathcal{C}$ converging to a limit $u$ and $\left(v_{n}\right)_{n \geq 1}$ a sequence such that for all $n, v_{n} \in \mathcal{T}\left(u_{n}\right)$ and converging to a limit $v$. We define, for all $n \in \mathbb{N}, p_{n}$ associated to $v_{n}$ in the sense of relation (3.1). We need to prove that $v \in \mathcal{T}(u)$.

Consider the system (2.8)-(2.11) to which we adjoin (2.28)-(2.29) with initial conditions (2.5) and (2.30), written for the $u_{n}$ :

$$
\begin{aligned}
S_{n}^{\prime}(t) & =\mu\left(1-\dot{u}_{n}(t)\right)-\beta S_{n}(t) I_{n}(t)-\mu S_{n}(t), S_{n}(0)=S_{0} \\
I_{n}^{\prime}(t) & =\left(\beta S_{n}(t)-\gamma-\mu\right) I_{n}(t), I_{n}(0)=I_{0} \\
R_{n}^{\prime}(t) & =\gamma I_{n}(t)-\mu R_{n}(t), R_{n}(0)=1-S_{n}(0)-I_{n}(0) \\
V_{n}^{\prime}(t) & =\mu \dot{u}_{n}(t)-\mu V_{n}(t), V_{n}(0)=0 \\
{\left[f_{I}^{u_{n}}(t)\right]^{\prime} } & =-\left(\mu+\beta I_{n}(t)\right) f_{I}^{u_{n}}(t), f_{I}^{u_{n}}(0)=1 \\
{\left[F_{I}^{u_{n}}(t)\right]^{\prime} } & =\mu f_{I}^{u_{n}}(t), F_{I}^{u_{n}}(0)=0 .
\end{aligned}
$$


This system is of the form $\dot{X}(t)=A(t)+\dot{u}(t) B(t)$ and has been studied in [11] where it is shown that is has a well defined meaning for $u_{n}, u \in L_{l o c}^{1}\left(\mathbb{R}_{+}\right)$. In particular for any finite horizon $T$ when $\left(u_{n}\right)_{n>1}$ converges in $L^{1}([0, T])$ to $u$ then the solution $S_{n}, \ldots, F_{I}^{u_{n}}(\cdot)$ (corresponding to $u_{n}$ ) converges pointwise to the solution $S, \ldots, F_{I}^{u}(\cdot)$ (corresponding to $u$ ).

On the other hand, one can see that $F_{I}^{u}(\tau)$ converges when $\tau \rightarrow \infty$ to $F_{I}^{u}(\infty)$ uniformly with respect to $u$ (their difference is bounded by $e^{-T \mu}$ ).

In conclusion since $\left(u_{n}\right)_{n \geq 1}$ converges to $u$ for the uniform convergence on compacts, we obtain by equation $(2.27)$ that $\phi_{I}^{u_{n}}(t)$ is converging to $\phi_{I}^{u}(t)$ for any fixed $t$. Let us consider two cases :

(1) If $\phi_{I}^{u}(t)<r$, then for some $\epsilon>0$ small enough there exists $n_{\epsilon}$ such that $\phi_{I}^{u_{n}}(t)<$ $r-\epsilon$ for all $n \geq n_{\epsilon}>0$. Remark now that $\phi_{I}^{u_{n}}(\cdot)$ are Lipschitz (with constant $C_{L}$ independent of $\left.u_{n}\right)$. Then on $\left.V_{\epsilon}=\right] t-\epsilon /\left(2 C_{L}\right), t+\epsilon /\left(2 C_{L}\right)\left[\right.$ we have $\phi_{I}^{u_{n}}(\cdot)<$ $r-\epsilon / 2<r$. In this neighborhood, and for $n \geq n_{\eta}, p_{n}(\cdot)=0$, which implies that $v_{n}(\cdot)=c s t$, thus $v(\cdot)=c s t$. Hence, $\dot{v}=0$ in the neighborhood $V_{\eta}$ and in particular $\dot{v}(t)=0$.

(2) We can prove, in the same way, that if $\phi_{I}^{u}(t)>r$, then $\dot{v}(t)=1$.

Hence $v \in \mathcal{T}(u)$.

Remark 3.4. The Theorem 3.1 only guarantees the existence of an equilibrium and not its uniqueness. Proving uniqueness requires in general more information on the problem (for instance convexity / monotony as in [44, 43, 34], which is not true in our cases, or analytic formulas as in [42]) and can break down in general (see [40]). Nevertheless in all situations we tested numerically, only one equilibrium was found.

\section{Numerical Simulations}

In order to find the Nash equilibrium, we need to minimize the cost of any individual; thus we need to simulate the model (2.1)-(2.4) but also the probability of infection $\phi_{I}^{u}(\cdot)$.

4.1. Approximation for a finite horizon time $T$. Note that, in the numerical approach, the time horizon, denoted $T$, is finite. Hence, in order to compute $F_{I}^{u}(\infty)$, we need to make an approximation; here we suppose that the population of infected is constant after $T$, that is for any $t \geq T, I^{u}(t) \simeq I^{u}(T)$. Under this assumption, we can 
estimate $F_{I}^{u}(\infty)$ in the following way :

$$
\begin{aligned}
F_{I}^{u}(\infty) & =\int_{0}^{\infty} \mu \exp \left[-\int_{0}^{\tau}\left(\mu+\beta I^{u}(s)\right) d s\right] d \tau \\
& \simeq \int_{0}^{T} \mu \exp \left[-\int_{0}^{\tau}\left(\mu+\beta I^{u}(s)\right) d s\right] d \tau \\
& +\int_{T}^{\infty} \mu \exp \left[-\int_{0}^{T}\left(\mu+\beta I^{u}(s)\right) d s\right] \times \exp \left[-\int_{T}^{\tau}\left(\mu+\beta I^{u}(T)\right) d s\right] d \tau \\
& =F_{I}^{u}(T)+\exp \left[-\int_{0}^{T}\left(\mu+\beta I^{u}(s)\right) d s\right] \times \int_{T}^{\infty} \mu e^{-\left(\mu+\beta I^{u}(T)\right)(\tau-T)} d \tau \\
& =F_{I}^{u}(T)+\mu f_{I}^{u}(T) \times \int_{T}^{\infty} e^{-\left(\mu+\beta I^{u}(T)\right)(\tau-T)} d \tau \\
& =F_{I}^{u}(T)+\mu f_{I}^{u}(T) \times \int_{0}^{\infty} e^{-\left(\mu+\beta I^{u}(T)\right) s} d s \\
& =F_{I}^{u}(T)+\frac{\mu f_{I}^{u}(T)}{\mu+\beta I^{u}(T)} .
\end{aligned}
$$

Hence we set :

$\phi_{I}^{u}(t)=1-\frac{1}{f_{I}^{u}(t)}\left(F_{I}^{u}(\infty)-F_{I}^{u}(t)\right) \simeq 1-\frac{1}{f_{I}^{u}(t)} \times\left[F_{I}^{u}(T)+\frac{\mu f_{I}^{u}(T)}{\mu+\beta I^{u}(T)}-F_{I}^{u}(t)\right]$.

Thus, to simulate the model (2.1)-(2.4) and the probability infection, we solve numerically the system (3.3)-(3.8) and apply (4.1). Moreover in order to improve the precision of the numerical solver, we sometimes rewrite the system (3.3)-(3.8) by replacing $I(t)$ by $\log (I(t))$ and solve the corresponding equivalent ODE.

4.2. Finding the fixed point. The result of the Section 3 guarantees the existence of at least one equilibrium. But this method is not constructive and has to be supplemented by a procedure to find the fixed point. The presentation of this procedure is the object of this section.

The procedure is iterative, denote by $n$ the iteration counter. For $n=0$, any $p \in \mathcal{P}$ can be chosen; for our numerical tests, we take $p^{0}(t)=p_{\text {csne }}$. Suppose now that we are at the iteration $n$. To $p^{n}$ we associate $u^{n}(\cdot)=\int_{0}^{b} p^{n}(s) d s$ and the probability of infection $\phi_{I}^{u^{n}}(\cdot)$ is calculated as indicated in section 4.1. As shown in the equation (2.32), the minimization problem of the cost of an individual born in $t$ is equivalent to:

$$
\min _{p(t) \in[0,1]}\left\{p(t)\left(r_{V}-r_{I} \phi_{I}^{u^{n}}(t)\right)\right\} .
$$

The specific of our procedure is that we do not take as next iterate the minimizer in the above term. Instead, in a way similar to gradient flows as implemented in [51], we ask that the transition from $p^{n}$ to $p^{n+1}$ be smooth; the intuitive interpretation is that the individual wants to minimize his/her cost but the new vaccination strategy need to be near the previous one. Hence, we minimize for all $t$ and for given parameter $\delta \tau>0$ :

$$
\min _{p(t) \in[0,1]}\left\{\frac{\left(p(t)-p^{n}(t)\right)^{2}}{2 \delta \tau}+p(t)\left(r_{V}-r_{I} \phi_{I}^{u^{n}}(t)\right)\right\} .
$$

The constant $\delta \tau$ plays the role of a pseudo-time; we denote $\tau_{n}=n \delta \tau$. If one forgets for a moment the restriction that $p \in \mathcal{P}$, when $\delta \tau \rightarrow 0$ the sequence of $p^{n}$ will approach a 
solution of the following equation set in the space of curves $\frac{\partial}{\partial \tau} p_{\tau}(t)+r_{V}-r_{I} \phi_{I}^{u_{\tau}(\cdot)}(t)=0$ (here $u_{\tau}$ is the primitive of $p_{\tau}$ ), see [55] for details.

The next iterate $p^{n+1}$ is the solution of the minimization problem (4.3):

$$
p^{n+1}(t)=\operatorname{proj}_{[0,1]}\left\{p^{n}(t)-\delta \tau\left(r_{V}-r_{I} \phi_{I}^{u^{n}}(t)\right)\right\},
$$

where $\operatorname{proj}_{[0,1]}(x)=\max (\min (x, 1), 0)$. Hence, we have a new vaccination function and the procedure iterates until a stopping criterion is met.

The stopping criterion is related to the incentive to vaccinate: given $p^{n}$ and $u^{n}$, one may ask whether a Nash equilibrium has been reached. Recall that a Nash equilibrium is a strategy such that there is no gain in changing it unilateraly. We define the best obtainable cost at any time $t$ by:

$$
\min _{p \in[0,1]}\left\{p r_{V}+(1-p) r_{I} \phi_{I}^{u^{n}}(t)\right\}=\min \left\{r_{V}, r_{I} \phi_{I}^{u^{n}}(t)\right\} .
$$

In other terms, the Nash equilibrium is reached when, for the given $u^{n}$ and any time $t$, the best obtainable cost defined in equation (4.4) equals the cost obtained with $p^{n}(t)$. Since the second cost is always larger than the first, a good stopping criterion is:

$$
E_{p^{n}}(t):=p^{n}(t) r_{V}+\left(1-p^{n}(t)\right) r_{I} \phi_{I}^{u^{n}}(t)-\min \left\{r_{V}, r_{I} \phi_{I}^{u^{n}}(t)\right\} .
$$

This will also be called the incentive to change strategy.

4.3. Results. We consider, in this section, an epidemic with the following characteristics: $\mu=1 / 80, R_{0}=15, \gamma=1000 \mu, \beta=R_{0} *(\gamma+\mu), r=0.1, T=80, S_{0}=6.53 \%$, $I_{0}=7.4 e-6$.

In this case the long-term, time independent, convergently stable Nash equilibrium is attained at a level of $p_{\text {csne }}=1-1 /\left(R_{0} *(1-r)\right)=0.9259$. We implement the procedure described in Section 4.2 (with $\delta \tau=0.1$ ) which converges as shown in figure 3, where the incentive to change strategies decreases by several orders of magnitude. We observe thus that a good solution has been obtained.

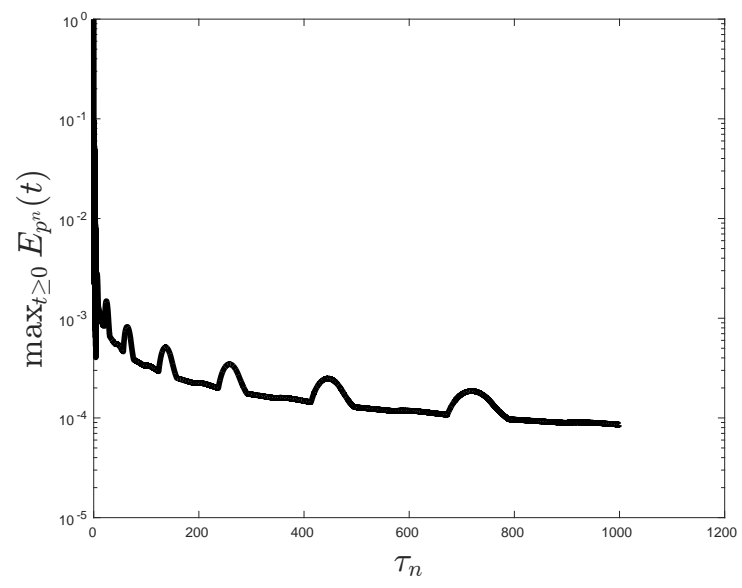

FiguRE 3. Decrease, with respect to $\tau$, of the incentive to change strategy as defined in equation (4.5). The maximum mismatch between the current cost and optimal cost decreases by several orders of magnitude and as such one can consider the solution attained.

The probability of vaccination $p(t)$, which is the main result of the simulation and which completely characterize the equilibrium, is given in figure 4 . Note that in order 
for the numerical simulation to complete, we had to choose a finite time horizon. So we have also checked that the solution is robust with respect to $T$. The comparison between two solutions, one with time horizon $T=20$ and another with $T=80$, is also presented in figure 4. Further numerical tests for $T=800$, not shown here, display similar behavior. The similarity between the solutions for $T=20$ and larger values of $T$ shows that the main dynamics happens already in the $T=20$ time horizon, which is more compatible with the time span of the individuals than the time-independent $p_{\text {csne }}$ vaccination strategy (see section 2 ).

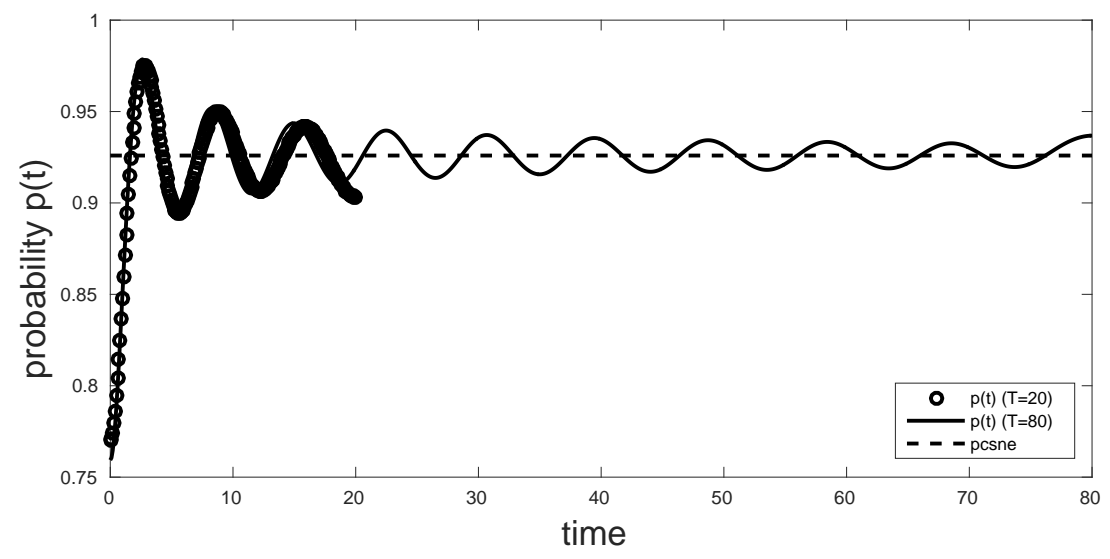

FIgURE 4 . The probability of vaccination (for $T=20$ and $T=80$ ).

We refer to figure 1 for the curve $t \mapsto(S(t), I(t))$.

Finally, we compare in figure 5 the quality of two equilibrium strategies: the $p(t)=$ $p_{\text {csne }}$ vaccination rate, which is an equilibrium within the class of time independent strategies (but not necessarily so in the class of time dependent vaccination rates) and the equilibrium strategy in figure 4 within the class of time dependent vaccination rates. The quality of each equilibrium is illustrated by plotting, for an individual born at $t=0$, the two terms of the incentive to change strategies (see equation (4.5)): in grey the cost $p(t) r_{V}+(1-p(t)) r_{I} \phi_{I}^{u}(t)$ experienced by the individual following the equilibrium strategy and in white the minimum possible cost $\min \left\{r_{V}, r_{I} \phi_{I}^{u}(t)\right\}$. The time dependent optimal strategy has lower incentive to change, resulting in a higher quality equilibrium.

\section{ACKNOWLEDGEMENTS}

G.T. acknowledges support from the Agence Nationale de la Recherche (ANR), projects EMAQS (ANR-2011-BS01-017-01), CINE-PARA and MFG (ANR-16-CE40-0015-01).

\section{Appendix A. Clake generalized gradients}

To recall the definition of the Clarke generalized gradients we follow the presentation in [16, section 10.1 page 194] and [17]. Let $X$ be a Banach space, $X^{*}$ its dual and $x \in X$; also take $f: X \rightarrow \mathbb{R}$ to be a functional which is Lipschitz with constant $L>0$ in a neighborhood of $x$, that is, for some $\epsilon>0$, we have $\|f(y)-f(z)\| \leq L\|y-z\|$ for all $y, z$ in the ball of center $x$ and radius $\epsilon$. The generalized directional derivative of $f$ at $x$ in the direction $v$, denoted $f^{o}(x ; v)$, is defined as

$$
f^{o}(x, v)=\limsup _{y \rightarrow x, t \downarrow 0} \frac{f(y+t v)-f(y)}{t} .
$$




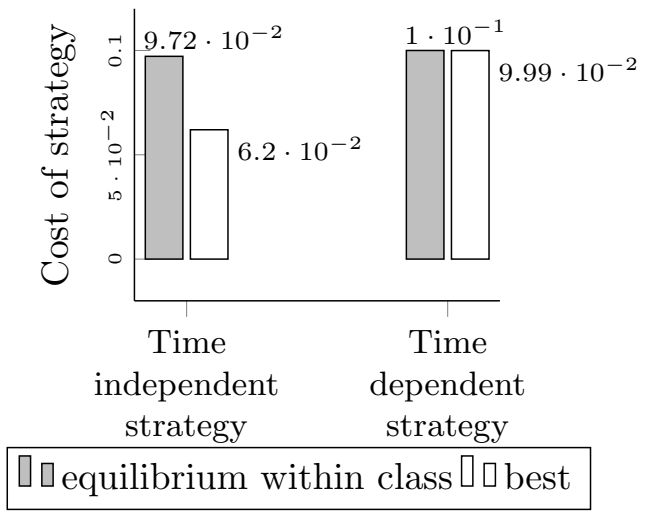

FIGURE 5. The comparison of the incentive to change the equilibrium strategy for the time dependent / independent classes. The parameters are as in figure 1 . The grey bar is always superior to the white bar and their difference is related to the quality of the equilibrium: smaller the difference higher the quality.

Note that $\left\|f^{o}(x, v)\right\| \leq L\|v\|$ for any $v \in X$; moreover, as function of $v$, the directional derivative $f^{o}(x, v)$ is subadditive i.e. $f^{o}(x, v+w) \leq f^{o}(x, v)+f^{o}(x, w), \forall v, w \in X$. In particular it can be lower bounded by a linear functional in $X^{*}$. The (Clarke) generalized gradient of $f$ at $x$ denoted $\bar{\partial} f(x)$ or $\dot{f}(x)$ is the set of all such linear functionals; the formal definition is the following:

$$
\bar{\partial} f(x)=\left\{\xi \in X^{*} \mid f^{o}(x, v) \geq\langle v, \xi\rangle, \forall v \in X\right\} .
$$

It can be shown that the Clarke generalized gradient is a non empty, convex, (weakly-*) compact subset of $X^{*}$. In particular when $X=\mathbb{R}^{k}$ for some $k \in \mathbb{N}^{*}, \bar{\partial} f(x)$ is the convex hull of the set $\left\{\lim _{\ell \rightarrow \infty} \nabla f\left(x_{\ell}\right)\right\}$ for any sequence $x_{\ell}$ converging to $x$ such that:

- $\nabla f\left(x_{\ell}\right)$ exists $\forall \ell$ (recall that since $f$ is Lipschitz it is differentiable a.e.) and

- the limit $\lim _{\ell \rightarrow \infty} \nabla f\left(x_{\ell}\right)$ exists.

\section{Appendix B. TeChNical Details CONCERning the PROBABILITy of INFECTION}

Recall that $\phi_{I}^{u}($.$) is a function from \mathbb{R}_{+}$to $[0,1]$ such that, for any $t \in \mathbb{R}_{+}, \phi_{I}^{u}(t)$ is the probability of infection during the life of an individual, born in $t$ and not vaccinated, when the population follows the vaccination strategy $u$. In mathematical terms, for any individual born in $t \geq 0$,

$$
\phi_{I}^{u}(t)=\mathbb{P}\left(\exists \tau \geq t \text { such that } M_{\tau}^{t}=I \mid M_{t}^{t}=S\right) .
$$

In order to compute $\phi_{I}^{u}(t)$ we introduce the probability $\varphi_{I}^{u, t}($.$) of infection before \tau$ :

$$
\varphi_{I}^{u, t}(\tau)=\mathbb{P}\left(\exists s \in[t, \tau] \text { such that } M_{s}^{t}=I \mid M_{t}^{t}=S\right) .
$$


Hence, we have :

$$
\begin{aligned}
\varphi_{I}^{u, t}(\tau+\Delta \tau) & =\mathbb{P}\left(M_{\tau+\Delta \tau}^{t} \in I \cup R \cup D_{2} \mid M_{t}^{t}=S\right) \\
& =\mathbb{P}\left(M_{\tau+\Delta \tau}^{t} \in I \cup R \cup D_{2} \mid M_{\tau}^{t}=S\right) \times \mathbb{P}\left(M_{\tau}^{t}=S \mid M_{t}^{t}=S\right) \\
& +\mathbb{P}\left(M_{\tau+\Delta \tau}^{t} \in I \cup R \cup D_{2} \mid M_{\tau}^{t}=I\right) \times \mathbb{P}\left(M_{\tau}^{t}=I \mid M_{t}^{t}=S\right) \\
& +\mathbb{P}\left(M_{\tau+\Delta \tau}^{t} \in I \cup R \cup D_{2} \mid M_{\tau}^{t}=R\right) \times \mathbb{P}\left(M_{\tau}^{t}=R \mid M_{t}^{t}=S\right) \\
& +\mathbb{P}\left(M_{\tau+\Delta \tau}^{t} \in I \cup R \cup D_{2} \mid M_{\tau}^{t}=D_{1}\right) \times \mathbb{P}\left(M_{\tau}^{t}=D_{1} \mid M_{t}^{t}=S\right) \\
& +\mathbb{P}\left(M_{\tau+\Delta \tau}^{t} \in I \cup R \cup D_{2} \mid M_{\tau}^{t}=D_{2}\right) \times \mathbb{P}\left(M_{\tau}^{t}=D_{2} \mid M_{t}^{t}=S\right),
\end{aligned}
$$

with

$$
\begin{aligned}
\mathbb{P}\left(M_{\tau+\Delta \tau}^{t} \in I \cup R \cup D_{2} \mid M_{\tau}^{t}=D_{1}\right) & =0 \\
\mathbb{P}\left(M_{\tau+\Delta \tau}^{t} \in I \cup R \cup D_{2} \mid M_{\tau}^{t}=I\right) & =1 \\
\mathbb{P}\left(M_{\tau+\Delta \tau}^{t} \in I \cup R \cup D_{2} \mid M_{\tau}^{t}=R\right) & =1 \\
\mathbb{P}\left(M_{\tau+\Delta \tau}^{t} \in I \cup R \cup D_{2} \mid M_{\tau}^{t}=D_{2}\right) & =1 \\
\mathbb{P}\left(M_{\tau+\Delta \tau}^{t} \in I \cup R \cup D_{2} \mid M_{\tau}^{t}=S\right) & =\beta I^{u}(\tau) \Delta \tau+o(\Delta \tau) .
\end{aligned}
$$

Hence,

$$
\begin{aligned}
\varphi_{I}^{u, t}(\tau+\Delta \tau) & =\beta I^{u}(\tau) \Delta \tau \times \mathbb{P}\left(M_{\tau}^{t}=S \mid M_{t}^{t}=S\right)+\mathbb{P}\left(M_{\tau}^{t} \in I \cup R \cup D_{2} \mid M_{t}^{t}=S\right)+o(\Delta \tau) \\
& =\beta I^{u}(\tau) \Delta \tau \times \mathbb{P}\left(M_{\tau}^{t}=S \mid M_{t}^{t}=S\right)+\varphi_{I}^{u, t}(\tau)+o(\Delta \tau) .
\end{aligned}
$$

We denote $r^{u, t}(\tau)=\mathbb{P}\left(M_{\tau}^{t}=S \mid M_{t}^{t}=S\right)$ the probability of staying susceptible between $t$ and $\tau$. We compute this probability :

$$
\begin{aligned}
r^{u, t}(\tau+\Delta \tau) & =\mathbb{P}\left(M_{\tau+\Delta \tau}^{t}=S \mid M_{\tau}^{t}=S\right) \times \mathbb{P}\left(M_{\tau}^{t}=S \mid M_{t}^{t}=S\right) \\
& =\left(1-\beta I^{u}(\tau) \Delta \tau-\mu \Delta \tau\right) r^{u, t}(\tau)+o(\Delta \tau) .
\end{aligned}
$$

Hence, the probability of staying susceptible between $t$ and $\tau$ is :

$$
r^{u, t}(\tau)=e^{-\mu(\tau-t)} \exp \left(-\int_{t}^{\tau} \beta I^{u}(s) d s\right)
$$

Hence,

$$
\varphi_{I}^{u, t}(\tau+\Delta \tau)=\beta I^{u}(\tau) \Delta \tau e^{-\mu(\tau-t)} \exp \left(-\int_{t}^{\tau} \beta I^{u}(s) d s\right)+\varphi_{I}^{u, t}(\tau)+o(\Delta \tau),
$$

which leads to

$$
\begin{aligned}
\frac{d \varphi_{I}^{u, t}(\tau)}{d \tau} & =\beta I^{u}(\tau) e^{-\mu(\tau-t)} \exp \left(-\int_{t}^{\tau} \beta I^{u}(s) d s\right) \\
& =e^{-\mu(\tau-t)} \exp \left(\int_{0}^{t} \beta I^{u}(s) d s\right) \times \beta I^{u}(\tau) \exp \left(-\int_{0}^{\tau} \beta I^{u}(s) d s\right) \\
& =-e^{-\mu(\tau-t)} \exp \left(\int_{0}^{t} \beta I^{u}(s) d s\right) \frac{d\left[\exp \left(-\int_{0}^{\tau} \beta I^{u}(s) d s\right)\right]}{d \tau} \\
& =-e^{-\mu(\tau-t)} \exp \left(\int_{0}^{t} \beta I^{u}(s) d s\right) \frac{d \psi_{I}^{u}(\tau)}{d \tau},
\end{aligned}
$$


by setting $\psi_{I}^{u}(\tau)=\exp \left(-\int_{0}^{\tau} \beta I^{u}(s) d s\right)$.

Finally, we just have to compute the probability of infection during the life of an individual born in $t$ who is not vaccinated, which is :

$$
\begin{aligned}
\phi_{I}^{u}(t) & =\int_{t}^{+\infty} d \varphi_{I}^{u, t}(\tau) \\
& =-\int_{t}^{+\infty} \exp \left(-\mu(\tau-t)+\int_{0}^{t} \beta I^{u}(s) d s\right)\left[\psi_{I}^{u}(\tau)\right]^{\prime} d \tau \\
& =-\exp \left(\mu t+\int_{0}^{t} \beta I^{u}(s) d s\right) \int_{t}^{+\infty} e^{-\mu \tau}\left[\psi_{I}^{u}(\tau)\right]^{\prime} d \tau
\end{aligned}
$$

By an integration by parts, we obtain :

$$
\phi_{I}^{u}(t)=1-\int_{t}^{+\infty} \mu \exp \left(-\int_{t}^{\tau}\left(\mu+\beta I^{u}(s)\right) d s\right) d \tau .
$$

In fact, this probability is solution of the differential equation :

$$
\begin{aligned}
\frac{d \phi_{I}^{u}(t)}{d t} & =\mu \exp \left(-\int_{t}^{t}\left(\mu+\beta I^{u}(s)\right) d s\right)-\int_{t}^{+\infty} \mu \frac{\partial}{\partial t}\left[\exp \left(-\int_{t}^{\tau}\left(\mu+\beta I^{u}(s)\right) d s\right)\right] d \tau \\
& =\mu-\mu \int_{t}^{+\infty}\left(\mu+\beta I^{u}(t)\right) \exp \left(-\int_{t}^{\tau}\left(\mu+\beta I^{u}(s)\right) d s\right) d \tau \\
& =\mu-\left(\mu+\beta I^{u}(t)\right)\left(1-\phi_{I}^{u}(t)\right)=\left(\mu+\beta I^{u}(t)\right) \phi_{I}^{u}(t)-\beta I^{u}(t) .
\end{aligned}
$$

In order to get an explicit form for $\phi_{I}^{u}(0)$, we define, for all $t \geq 0$ :

$$
\begin{aligned}
f_{I}^{u}(t) & =\exp \left[-\int_{0}^{t}\left(\mu+\beta I^{u}(\tau)\right) d \tau\right] \\
F_{I}^{u}(t) & =\int_{0}^{t} \mu f_{I}^{u}(\tau) d \tau \\
\mathcal{F}_{I}^{u}(t) & =1-\phi_{I}^{u}(t) .
\end{aligned}
$$

The last function satisfies the following differential equation :

$$
\begin{aligned}
{\left[\mathcal{F}_{I}^{u}(t)\right]^{\prime} } & =-\mu+\left(\mu+\beta I^{u}(t)\right) \mathcal{F}_{I}^{u}(t) \\
\mathcal{F}_{I}^{u}(0) & =\int_{0}^{+\infty} \mu \exp \left(-\int_{0}^{\tau}\left(\mu+\beta I^{u}(s)\right) d s\right) d \tau \\
& =\int_{0}^{+\infty} \mu f_{I}^{u}(\tau) d \tau=F_{I}^{u}(\infty)
\end{aligned}
$$

Note that :

$$
\left[\mathcal{F}_{I}^{u}(t) \times f_{I}^{u}(t)\right]^{\prime}=\left\{\left[\mathcal{F}_{I}^{u}(t)\right]^{\prime}-\mathcal{F}_{I}^{u}(t)\left(\mu+\beta I^{u}(t)\right)\right\} \times f_{I}^{u}(t)=-\mu f_{I}^{u}(t)
$$

Thus, $\mathcal{F}_{I}^{u}(t) \times f_{I}^{u}(t)=\mathcal{F}_{I}^{u}(0)-\int_{0}^{t} \mu f_{I}^{u}(\tau)$ and therefore

$$
\mathcal{F}_{I}^{u}(t)=\frac{1}{f_{I}^{u}(t)}\left[F_{I}^{u}(\infty)-F_{I}^{u}(t)\right]
$$

Hence, for all $t \geq 0$, we obtain :

$$
\phi_{I}^{u}(t)=1-\frac{F_{I}^{u}(\infty)-F_{I}^{u}(t)}{f_{I}^{u}(t)}, \phi_{I}^{u}(0)=1-F_{I}^{u}(\infty) .
$$




\section{REFERENCES}

[1] Andris Abakuks. Optimal immunisation policies for epidemics. Advances in Appl. Probability, 6:494$511,1974$.

[2] Sudhir Anand and Kara Hanson. Disability-adjusted life years: a critical review. Journal of Health Economics, 16(6):685 - 702, 1997.

[3] R. M. Anderson and R. M. May. Infectious Diseases of Humans Dynamics and Control. Oxford University Press, 1992.

[4] Nicolas Bacaër. A short history of mathematical population dynamics. Springer-Verlag London, Ltd., London, 2011.

[5] Fan Bai. Uniqueness of nash equilibrium in vaccination games. Journal of Biological Dynamics, 10(1):395-415, 2016. PMID: 27465224.

[6] Chris T. Bauch and David J. D. Earn. Vaccination and the theory of games. Proc. Natl. Acad. Sci. USA, 101(36):13391-13394 (electronic), 2004.

[7] Chris T. Bauch, Alison P. Galvani, and David J. D. Earn. Group interest versus self-interest in smallpox vaccination policy. Proceedings of the National Academy of Sciences, 100(18):10564-10567, 2003.

[8] C.T. Bauch. Imitation dynamics predict vaccinating behaviour. Proc Biol Sci, 272(1573):1669-1675, 2005.

[9] Alain Benoussan, Jens Frehse, and Phillip Yam. Mean Field Games and Mean Field Type Control Theory. Springer-Verlag New York, 2013.

[10] Romulus Breban, Raffaele Vardavas, and Sally Blower. Mean-field analysis of an inductive reasoning game: Application to influenza vaccination. Phys. Rev. E, 76:031127, Sep 2007.

[11] A. Bressan, JR., and F. Rampazzo. Impulsive control systems with commutative vector fields. $J$. Optim. Theory Appl., 71(1):67-83, 1991.

[12] Dagobert L. Brito, Eytan Sheshinski, and Michael D. Intriligator. Externalities and compulsary vaccinations. Journal of Public Economics, 45(1):69 - 90, 1991.

[13] Bruno Buonomo, Alberto d'Onofrio, and Deborah Lacitignola. Global stability of an \{SIR $\}$ epidemic model with information dependent vaccination. Mathematical Biosciences, 216(1):9 - 16, 2008.

[14] Frederick H. Chen. A susceptible-infected epidemic model with voluntary vaccinations. Journal of Mathematical Biology, 53(2):253-272, 2006.

[15] Frederick H. Chen. Modeling the effect of information quality on risk behavior change and the transmission of infectious diseases. Mathematical Biosciences, 217(2):125 - 133, 2009.

[16] Francis Clarke. Functional analysis, calculus of variations and optimal control, volume 264 of Graduate Texts in Mathematics. Springer, London, 2013.

[17] Frank H. Clarke. Generalized gradients and applications. Trans. Am. Math. Soc., 205:247-262, 1975.

[18] Claudia T. Codeço, Paula M. Luz, Flavio Coelho, Alison P Galvani, and Claudio Struchiner. Vaccinating in disease-free regions: a vaccine model with application to yellow fever. Journal of The Royal Society Interface, 4(17):1119-1125, 2007.

[19] Flávio Codeço Coelho and Claudia T. Codeço. Dynamic modeling of vaccinating behavior as a function of individual beliefs. PLoS Comput Biol, 5(7):e1000425, 072009.

[20] Monica-Gabriela Cojocaru. Dynamic equilibria of group vaccination strategies in a heterogeneous population. Journal of Global Optimization, 40(1-3):51-63, 2008.

[21] Monica-Gabriela Cojocaru, ChrisT. Bauch, and MatthewD. Johnston. Dynamics of vaccination strategies via projected dynamical systems. Bulletin of Mathematical Biology, 69(5):1453-1476, 2007.

[22] O. Diekmann and J.A.P. Heesterbeek. Mathematical epidemiology of infectious diseases. Model building, analysis and interpretation. Wiley Series in Mathematical and Computational Biology. Chichester: Wiley., 1999.

[23] Alberto d'Onofrio, Piero Manfredi, and Ernesto Salinelli. Vaccinating behaviour, information, and the dynamics of SIR vaccine preventable diseases. Theoretical Population Biology, 71(3):301 - 317, 2007.

[24] Alberto d'Onofrio, Piero Manfredi, and Ernesto Salinelli. Fatal SIR diseases and rational exemption to vaccination. Mathematical Medicine and Biology, 25(4):337-357, 2008.

[25] Ky Fan. Fixed-point and minimax theorems in locally convex topological linear spaces. Proc. Natl. Acad. Sci. USA, 38:121-126, 1952. 
[26] Paul E. M Fine and Jacqueline A Clarkson. Individual versus public priorities in the determination of optimal vaccination policies. American Journal of Epidemiology, 124(6):1012-1020, 1986.

[27] Peter J. Francis. Optimal tax/subsidy combinations for the flu season. Journal of Economic Dynamics and Control, 28(10):2037 - 2054, 2004.

[28] Drew Fudenberg and Jean Tirole. Game theory. MIT Press, Cambridge, MA, 1991.

[29] Eriko Fukuda, Satoshi Kokubo, Jun Tanimoto, Zhen Wang, Aya Hagishima, and Naoki Ikegaya. Risk assessment for infectious disease and its impact on voluntary vaccination behavior in social networks. Chaos, Solitons 8 Fractals, 68:1 - 9, 2014.

[30] Sebastian Funk, Marcel Salathé, and Vincent A. A. Jansen. Modelling the influence of human behaviour on the spread of infectious diseases: a review. Journal of The Royal Society Interface, $7(50): 1247-1256,2010$.

[31] Alison P. Galvani, Timothy C. Reluga, and Gretchen B. Chapman. Long-standing influenza vaccination policy is in accord with individual self-interest but not with the utilitarian optimum. Proceedings of the National Academy of Sciences, 104(13):5692-5697, 2007.

[32] Pierre-Yves Geoffard and Tomas Philipson. Disease eradication: Private versus public vaccination. The American Economic Review, 87(1):pp. 222-230, 1997.

[33] I.L. Glicksberg. A further generalization of the Kakutani fixed point theorem, with application to Nash equilibrium points. Proc. Am. Math. Soc., 3:170-174, 1952.

[34] Diogo A. Gomes, Joana Mohr, and RafaelRigão Souza. Continuous time finite state mean field games. Applied Mathematics \& Optimization, 68(1):99-143, 2013.

[35] Olivier Guéant, Jean-Michel Lasry, and Pierre-Louis Lions. Mean field games and applications. In Paris-Princeton Lectures on Mathematical Finance 2010, volume 2003 of Lecture Notes in Math., pages 205-266. Springer, Berlin, 2011.

[36] Herbert W. Hethcote and Paul Waltman. Optimal vaccination schedules in a deterministic epidemic model. Mathematical Biosciences, 18(3-4):365-381, December 1973.

[37] Minyi Huang, Roland P. Malhamé, and Peter E. Caines. Nash equilibria for large-population linear stochastic systems of weakly coupled agents. In Elkébir Boukas and Roland P. Malhamé, editors, Analysis, Control and Optimization of Complex Dynamic Systems, pages 215-252. Springer US, 2005.

[38] Minyi Huang, Roland P. Malhamé, and Peter E. Caines. Large population stochastic dynamic games: closed-loop mckean-vlasov systems and the Nash certainty equivalence principle. Commun. Inf. Syst., 6(3):221-252, 2006.

[39] John L. Kelley. General topology. 2nd ed. Springer-Verlag: New York - Heidelberg - Berlin, 1975.

[40] Aime Lachapelle, Julien Salomon, and Gabriel Turinici. Computation of mean field equilibria in economics. Math. Models Methods Appl. Sci., 20(4):567-588, 2010.

[41] Laetitia Laguzet and Gabriel Turinici. Global optimal vaccination in the SIR model: Properties of the value function and application to cost-effectiveness analysis. Mathematical Biosciences, 263:180 - 197, 2015.

[42] Laetitia Laguzet and Gabriel Turinici. Individual vaccination as Nash equilibrium in a SIR model with application to the 2009-2010 influenza A (H1N1) epidemic in France. Bulletin of Mathematical Biology, 77(10):1955-1984, 2015.

[43] Jean-Michel Lasry and Pierre-Louis Lions. Jeux à champ moyen. I: Le cas stationnaire. C. R., Math., Acad. Sci. Paris, 343(9):619-625, 2006.

[44] Jean-Michel Lasry and Pierre-Louis Lions. Jeux à champ moyen. II: Horizon fini et contrôle optimal. C. R., Math., Acad. Sci. Paris, 343(10):679-684, 2006.

[45] Jean-Michel Lasry and Pierre-Louis Lions. Mean field games. Japanese Journal of Mathematics, 2(1):229-260, 2007.

[46] Piero Manfredi and Alberto d'Onofrio. Modeling the interplay between human behavior and the spread of infectious diseases. Springer-Verlag New York, 2013.

[47] R. Morton and K. H. Wickwire. On the optimal control of a deterministic epidemic. Advances in Appl. Probability, 6:622-635, 1974.

[48] Johannes Müller. Optimal vaccination strategies-for whom? Mathematical biosciences, 139(2):133-154, 1997.

[49] Timothy C. Reluga, Chris T. Bauch, and Alison P. Galvani. Evolving public perceptions and stability in vaccine uptake. Math. Biosci., 204(2):185-198, 2006.

[50] Timothy C. Reluga and Alison P. Galvani. A general approach for population games with application to vaccination. Mathematical Biosciences, 230(2):67 - 78, 2011. 
[51] Francesco Salvarani and Gabriel Turinici. Optimal individual strategies for influenza vaccines with imperfect efficacy and limited persistence. working paper or preprint, April 2016.

[52] Franco Sassi. Calculating QALYs, comparing QALY and DALY calculations. Health Policy and Planning, 21(5):402-408, 2006.

[53] Suresh P. Sethi and Preston W. Staats. Optimal control of some simple deterministic epidemic models. J. Oper. Res. Soc., 29(2):129-136, 1978.

[54] Eunha Shim, Gretchen B. Chapman, Jeffrey P. Townsend, and Alison P. Galvani. The influence of altruism on influenza vaccination decisions. Journal of The Royal Society Interface, 9(74):22342243, September 2012.

[55] G. Turinici. Equilibrium flows on metric spaces. in preparation, 2017.

[56] Raffaele Vardavas, Romulus Breban, and Sally Blower. Can influenza epidemics be prevented by voluntary vaccination? PLoS Comput Biol, 3(5):e85, 052007.

[57] Zhen Wang, Chris T. Bauch, Samit Bhattacharyya, Alberto d'Onofrio, Piero Manfredi, Matjaž Perc, Nicola Perra, Marcel Salathé, and Dawei Zhao. Statistical physics of vaccination. Physics Reports, 664:1 - 113, 2016.

[58] Richard Zeckhauser and Donald Shepard. Where now for saving lives? Law and Contemporary Problems, 40:5-45, 1976.

E.H.: Université Paris-Dauphine, PSL Research University, 75016 PARIS, FRANCE

E-mail address: emma.hubert@dauphine.eu

G.T.: Université Paris-Dauphine, PSL Research University, CNRS UMR 7534, CERE-

MADE, 75016 PARIS, FRANCE \& Institut Universitaire De France

E-mail address: gabriel.turinici@dauphine.fr 